Relations industrielles

Industrial Relations

\title{
Résolutions des congrès ouvriers
} La démocratie ouvrière en oeuvre

\section{Jean Gagné}

Volume 3, numéro 2, octobre 1947

URI : https://id.erudit.org/iderudit/1023566ar

DOI : https://doi.org/10.7202/1023566ar

Aller au sommaire du numéro

Éditeur(s)

Département des relations industrielles de l’Université Laval

ISSN

0034-379X (imprimé)

1703-8138 (numérique)

Découvrir la revue

Citer cet article

Gagné, J. (1947). Résolutions des congrès ouvriers : la démocratie ouvrière en oeuvre. Relations industrielles / Industrial Relations, 3(2), 22-26.

https://doi.org/10.7202/1023566ar

Tous droits réservés @ C Département des relations industrielles de l’Université Laval, 1947
Ce document est protégé par la loi sur le droit d'auteur. L'utilisation des services d'Érudit (y compris la reproduction) est assujettie à sa politique d'utilisation que vous pouvez consulter en ligne.

https://apropos.erudit.org/fr/usagers/politique-dutilisation/ 
fluence morale qu'il possède sur les non-adhérents sont aussi des éléments d'appréciation dont il y a lieu de tenir le plus grand compte. En vertu de ce critère, il serait difficile de contester à l'une ou l'autre de nos trois centrales syndicales le caractère d'organisation représentative. Mais sur le plan de l'industrie, c'est-à-dire si l'on envisage les fédérales professionnelles, la question est plus compliquée.

Dans chaque cas où la représentation des organisations professionnelles des travailleurs se pose, plusieurs critères entrent donc en ligne de compte. Comment établir une juste proportion entre ces critères ? N'appartient-il pas aux organisations syndicales elles-mêmes de résoudre le problème avant que les pouvoirs publics soient appelés à le trancher ? Déjà des cartels syndicaux ont été constitués pour la négociation de con. ventions collectives de travail. Ne devrait-il pas en être ainsi au sujet de la représentation ?

Dans ce cas comme dans beaucoup d'autres, l'entente préalable et l'unité de représentation volontaire sont nettement préférables au maquis de la procédure.

\title{
Résalutions des congrès anuriers
}

\section{LA DÉMOCRATIE OUVRIÈRE EN OEUVRE}

\author{
Jean GAGNE
}

A chaque année, à peu près à la même époque, les grandes confédérations canadiennes d'unions ouvrières ont leurs assises générales en quelqu'endroit du pays. A ces conventions sont adoptées des résolutions qui deviennent leur programme d'action pour l'année à venir. Dans ces résolutions, on peut distinguer les tendances générales de ces unions et le sens de leurs prochaines revendications.

Le Congrès des Métiers et du Travail du Canada tenait sa 62ième Conférence dans la ville de Hamilton, le 24 septembre dernier. D'après les dernières statistiques, le Congrès compte 356,121 membres répartis en 2,536 syndicats locaux. ${ }^{1} \mathrm{La}$ Confédération des travailleurs catholiques du Canada a tenu la sienne à St-Hyacinthe du 14 au 18 septembre. D'après les mêmes statistiques, la Confédération compte 70,367 membres répartis en 338 sections locales, la plupart dans la Province de Québec. ${ }^{2}$ Voici un compte-rendu succinct de plusieurs centaines de résolutions que les délégués y ont adoptées.

\section{Mesures syndicales}

Dans ce domaine, quoique l'idée de sécurité syndicale semble être la même pour les deux groupements, les résolutions prennent cependant un caractère différent sans doute à cause du fait que le Congrès des métiers et du travail est une organisation plutôt à caractère national tandis que la C.T.C.C. en est une à caractère plus spécifique-
Ce que pensent deux grands groupements ouvriers sur des questions d'intérêt syndical, social et d'ordre général.

ment provincial. ${ }^{3} \mathrm{Ce}$ double caractère oriente sans nul doute le sens de leurs résolutions.

Le Congrès veut obtenir une \&loi qui interdise le recours aux injonctions pour empêcher les unions ouvrières légales de déclarer des grèves et de faire du piquetage et qui défende d'employer des briseurs de grève. »

Il décide d'aider les instituteurs à obtenir de meilleurs salaires et des conditions de travail améliorées, à l'avantage des instituteurs eux-mêmes et pour fournir des facilités d'instruction convenables aux enfants du pays.

On y trouve aussi une protestation énergique contre le contrôle par le gouvernement du vote pour ou contre la grève, comme disparition finale des unions ouvrières libres et immixtion du gouvernement dans la régie interne du travail organisé, ainsi qu'une demande pour exempter les cotisations ouvrières comme dépenses aux fins de l'impôt sur le revenu. D'autre part, on veut le rétablissement de la taxe sur les excédents de bénéfices « non seulement pour faire porter le fardeau par ceux qui sont le plus en état de le porter, mais

(1) Communiqué, ministère du Travail, Ottawa, le 11 septembre 1947.

(2) Le congrès à Hamilton, Le monde ouvrier, Montréal, septembre 1947. Le travail, Québec, octobre 1947.

(3) En effet, le Congrès syndical ouvrier canadien compte 356,121 membres dispersés dans les neuf provinces du Canada. La C.T.C.C. 70,367 membres presque tous dans la province de Québec. Or les syndiqués de la province de Québec sont au nombre de 208,546 membres appartenant à quatre grandes organisations ouvrières. 
aussi pour financer les mesures nationales du temps de paix. »

La C.T.C.C. a orienté ses demandes vers le domaine légal en proposant des améliorations aux lois du Travail.

D'abord elle exige l'application intégrale « sans favoritisme d'aucune sorte 》 de la loi fédérale de l'Assurance-chômage, sa modification afin de pouvoir accorder des crédits aux chômeurs, pour leur permettre de suivre des cours dans les différentes écoles de métiers.

Elle réclame la nomination de cinq fonctionnaires de l'Assurance-chômage dans le Québec et l'établissement de cinq bureaux d'adjudication des prestations d'après les différentes régions industrielles.

Et on demande le paiement de ces prestations après trois jours au lieu de neuf jours, après 160 jours de contribution au lieu de 180 jours, et l'on veut qu'elles soient augmentées proportionnellement à l'augmentation du coût de la vie.

D'après la C.T.C.C., la Commission devrait donner immédiatement leur livret d'assurancechômage aux ouvriers de la construction qui quittent leur travail, de sorte qu'ils ne soient pas obligés d'attendre 7 jours avant de recevoir leur livret pour pouvoir travailler ailleurs.

Il y aura aussi intervention auprès de l'Office National du Film pour qu'on réalise des films pratiques sur l'Assurance-chômage, nécessaires pour l'éducation du peuple canadien.

Quant aux Comités Paritaires, la C.T.C.C. étudie avec soin la constitution et les activités de la Fédération de ces comités afin de sauvegarder les droits des ouvriers organisés.

Demande est faite au ministre du Travail de faire une propagande pour renseigner les intéressés et l'opinion publique sur les comités paritaires en expliquant sa formation, son rôle et les services qu'il rend.

Ensuite, la C.T.C.C. propose toute une série d'amendements aux différentes lois du Travail, aux lois de Relations ouvrières, de la Convention collective, du Salaire minimum et des établissements industriels. Ces amendements ont pour but en général d'élargir le champ d'application de ces lois, les adapter aux conditions nouvelles de vie et de travail. Le but principal de ces derniers est de diminuer les délais des procédures, obtenir la représentation ouvrière dans toutes les commissions gouvernementales pour augmenter l'efficacité de ces lois et ainsi rendre justice plus complète aux ouvriers et aux chefs d'entreprise.

\section{Mesures de sécurité sociale}

Les deux grandes organisations ouvrières s'entendent pour favoriser linstruction dans le milieu ouvrier. Le Congrès des métiers et du travail propose au gouvernement fédéral d'établir un fonds pour venir en aide aux étudiants qui veulent poursuivre des études universitaires. La C.T.C.C. demande de payer des allocations familiales aux enfants âgés de plus de $\mathbf{1 6}$ ans qui continuent leurs études.

Elles s'entendent aussi sur la nécessité de régler la crise du logement ouvrier; le Congrès par un programme de construction de logements pouvant être loués à bon marché, financé par le crédit national et dans le but de loger dans des conditions convenables, les vétérans et ceux qui n'ont qu'un revenu modeste; la C.T.C.C. par la création d'un Crédit Ouvrier pour permettre à toutes les familles ouvrières d'accéder à la propriété d'une maison. Ce groupement affirme que c'est là le vrai remède dans la lutte contre toute doctrine subversive et annonce qu'il lancera une vaste campagne pour obtenir une loi nationale du logement plus humaine, i.e. moins capitaliste. On demande aussi au gouvernement fédéral de réorganiser dans ce but la Wartime Housing Enterprise de même que l'entreprise des anciens combattants.

La politique de ces organisations ouvrières concorde aussi sur la question des pensions. Le Congrès, lui, demande le paiement de pensions à 60 ans à toutes les personnes du sexe masculin, que leurs femmes aient droit à la pension en mème temps et que les autres personnes du sexe féminin y aient droit à 55 ans, sans déductions à cause d'autres revenus, et que le montant soit de $\$ 60.00$ plus le boni du coût de la vie. On demande aussi que cette pension s'applique aux aveugles à l'âge de 18 ans et que la loi s'applique à toute personne souffrant d'incapacité totale. La C.T.C.C. veut faire augmenter les versements aux mères nécessiteuses jusqu'au montant de $\$ 50.00$ par mois et si elle vit avec son époux invalide, jusqu'à $\$ 75.00$ par mois. Ce dernier groupe demandera aussi que - la pension des enfants soit portée de $\$ 5.00$ à $\$ 6.00$ par mois et qu'il soit payé une pension aux invalides sur le même principe que la pension aux aveugles.

Les deux groupements ouvriers ont aussi des résolutions semblables à propos de Radio-Canada. Le Congrès se déclare en faveur du maintien de cette Commission du gouvernement, avec un contrôle absolu sur l'émission de permis pour l'établissement de stations et demande au gouvernement de nommer un représentant du travail orga- 
nisé à la commission de radio-diffusion. La C.T.C.C. réitère sa demande pour que Radio-Canada exerce un contrôle efficace afin d'éliminer toute propagande subversive tant dans le propre réseau des postes de Radio-Canada que les postes privés sous sa surveillance.

On trouve en outre dans les demandes du Congrès, une suggestion à l'effet d'établir un système de rentes viagères aux vieillards avec contributions versées par les employés et les patrons, les frais d'administration étant à la charge du gouvernement; la demande d'une bibliothèque nationale qui puisse se comparer favorablement à celle d'aucun autre pays de la grandeur et de l'importance du Canada.

La C.T.C.C. a des résolutions de très grand intérêt sur les allocations familiales et limpôt sur le revenu. Elle demande qu'un taux égal soit accordé à tous les enfants sans égard au nombre, que les allocations familiales en pourcentage correspondent à l'augmentation du coût de la vie. Elle réitèrera ses pressions pour que les ouvriers mariés gagnant moins de $\$ 3,000$. par année et que les célibataires ayant un salaire inférieur à $\$ 2,000$. soient exemptés de l'impôt sur le revenu. Elle demande en outre l'abolition de l'usage des formules d'impôts pour tous ces ouvriers, sauf les cas de réclamations, que les allocations familiales ne soient pas taxables, ainsi que l'exemption $100 \%$ des frais médicaux sur le revenu global avant de prélever l'impôt. Elle propose ensuite trois amendements à la loi de l'impôt sur le revenu dans le sens suivant: - le gouvernement devrait faire toute réclamation de l'impôt sur le revenu d'un salarié dans les 12 mois suivant la déposition du rap. port, si la réclamation est faite après ce délai qu'elle ne comporte aucun intérêt (ce serait la pénalité du département.) Enfin, si le département de l'impôt ne fait pas un remboursement d'impôt dans ce même délai, qu'il soit accordé au contribuable le même intérêt que si ces contribuables étaient en défaut.

Le point le plus nouveau et le plus saillant de sécurité sociale soulevé dans les résolutions du Congrès est certes celui de l'adoption immédiate d'un système national d'assurance-santé avec aide financière aux municipalités pour la construction d'hôpitaux modernes; indemnité en argent pour maintenir le revenu pendant la maladie; l'intégration des soins curatifs et préventifs, ainsi que l'emploi du personnel nécessaire pour assurer à tous le maximum d'avantages aux points de vue médical et dentaire; la représentation ouvrière dans tous les conseils d'hôpitaux; et que toutes les personnes bénéficiant du système soient libres de choisir n'im- porte quels services curatifs personnels.

Le Congrès va jusqu'à demander la tenue d'une enquête nationale sur les conditions de travail des employés d'hôpitaux et «sur l'état de chose peu satisfaisant qui existe pour les jeunes femmes qui s'entraînent à la profession de gardemalade.»

On demande aussi aux autorités fédérales d'adopter une loi «établissant une assurance sociale avec service de médecine par l'Etat. » Il fut aussi décidé «qu'en attendant cette loi, le Congrès préparera un plan qui sera présenté au gouvernement fédéral, vu que le travailleur moyen est incapable de se procurer pour lui et sa famille, des soins médicaux suffisants. »

\section{Mesures d'intérêt général}

Les deux grandes organisations ouvrières ont encore ici des voeux communs. A propos de lindice du coût de la vie, par exemple, le Congrès demande de préparer un nouvel indice du coût de la vie basé sur les conditions et standards de vie actuels, ou sinon qu'on discontinue le présent coût de la vie; tandis que le bureau confédéral de la C.T.C.C. entend faire une étude complète et adéquate du coût de la vie et du budget familial et du problème du travail féminin.

C'est alors que la C.T.C.C. fait une suggestion tout à fait originale et qui laisse voir une tendance vers une forme nouvelle d'économie: elle réclame la formation d'une Commission Arbitrale des Prix aux fins de connaître et d'apprécier les motifs invoqués par les manufacturiers justifiant l'augmentation des prix de leurs produits ou de leurs services. L'organisation administrative en serait décentralisée et les tribunaux, distribués dans toutes les provinces canadiennes, entendraient les requêtes des augmentations de prix. Ces derniers tribunaux seraient composés de représentants des employeurs, des syndicats ouvriers, du gouvernement, des coopératives et des consommateurs. Les décisions de ces tribunaux, apprenant les motifs des augmentations des prix, seraient publiées par les services d'information des ministères provinciaux et fédéral du Travail.

Tous les employeurs qui ont augmenté le prix de leurs produits, de leurs services depuis Y'abolition des contrôles, et tous ceux qui, à lavenir, dèsireraient augmenter leurs prix, devraient soumettre leurs raisons justifiant l'augmentation désirée, à cette Commission Arbitrale des Prix el attendre sa décision avant d'ajuster leurs prix.

La C.T.C.C. réclame une enquête royale dans tout le Canada concernant la plaie du marché noir 
et fera des pressions auprès du gouvernement pour que celles-ci suppriment le marché noir des matériaux de construction. On propose encore l'augmentation des allocations familiales en pourcentage correspondant du coût de la vie.

On trouve aussi dans le rapport des deux conventions des résolutions communes sur limmigration. Le Congrès demande que le nombre des immigrants dépende de l'expansion industrielle et économique et de la situation qui existe au Canada pour ce qui est du chômage. La C.T.C.C. souligne que le gouvernement devrait apporter une solution adéquate au problème du logement avant de penser à faire entrer des immigrants au pays. Le Congrès note encore que la règle à suivre dans la colonisation des centres urbains et ruraux doit être de ne pas nuire au niveau de vie ou au bienêtre de notre population canadienne actuelle. Enfin, on se déclare opposé à une politique d'immigration qui accepterait «la venue au pays d'une main-d'oeuvre sous contrat et qui travaillerait en des conditions de servage.»

Le problème du lait a aussi intéressé les congrès ouvriers. Le Congrès des métiers et dı travail veut faire pression sur tous les gouvernements provinciaux de ce pays qui n'ont pas la pasteurisation du lait, pour les amener à passer une loi à cet effet. La C.T.C.C. elle, prie le gouvernement provincial de Québec d'instituer une enquête pour découvrir les responsables des augmentations prodigieuses du coût du lait et d'apporter sans délai un remède à cette augmentation.

Il est en outre proposé par le Congrès divers plans d'entreprises publiques pour parer à la crise qui approche. On pourrait en profiter pour donner au pays les améliorations dont il a tant besoin.

Le Congrès a aussi une résolution pour féliciter les autorités d'avoir passé la loi du Support des Prix des pêcheries et en même temps demande à la Commission de faire tout en son pouvoir pour traiter les pêcheurs avec justice sous tous les rapports surtout en leur garantissant un revenu annuel de $\$ 1,800$.

On demande en plus une loi provinciale uniforme pour réglementer l'enregistrement, la circulation et la responsabilité des cyclistes et on trouve que le même gouvernement devrait passer une loi défendant aux camions de stationner sur le bord des routes publiques quelles qu'elles soient, dans l'obscurité où la mi-obscurité vu que la plupart des accidents de la route sont dus à des stationnements de camions.

La C.T.C.C. fait encore quelques demandes d'intérêt général tant à la législature fédérale que provinciale. Au fédéral, elle s'oppose à toute cons. cription militaire des citoyens du Canada, en temps de paix comme en temps de guerre, vu qu'elle réprouve la participation de notre pays aux guerres extérieures et impériales, désire que soient déclarés hors la loi tout parti ou groupement conmuniste ainsi que les Témoins de Jéhovah, réclame la reconnaissance officielle du chant « O Canada » comme hymne national et fera des représentations pour que le choix du drapeau soit celui de la Ligue du Drapeau Canadien.

Dans le domaine provincial québécois, elle réclame que les fêtes religieuses d'obligation soient décrétées chômées, renouvelle ses instances pour que dans notre province, la loi du Dimanche soit observée, s'oppose à l'établissement. de nouvelles villes fermées, demande au Département de l'Instruction Publique d'introduire l'enseignement de la doctrine sociale de l'Eglise dans les cours primaires en vue de promouvoir l'éducation sociale chrétienne chez les adultes.

Et prie les autorités compétentes de voir à obtenir une plus grande surveillance dans les salles publiques où se donnent des vues animées ou autres spectacles pour les jeunes à la mi-obscurité, par exemple dans les stades de luttes ou autres endroits publics.

Enfin, elle déclare appuyer fortement les voeux que la J.O.C. a formulés lors de son grand congrès international récemment tenu à Montréal. En voici les principaux points:

a-La semaine de 40 heures.

b-L'orientation professionnelle généralisée dès la sortie de l'école afin de permettre aux jeunes de se choisir un métier selon leurs aptitudes et leurs goûts.

c-L'apprentissage obligatoire durant les heures de travail avec un plan semblable à celui des vétérans dit TRAINING ON THE JOB, en vertu duquel, l'employeur paie une partie du salaire et le gouvernement l'autre partie.

d-L'abolition du travail de nuit pour les femmes et les jeunes filles, ainsi que l'abolition de tout travail le dimanche. Des pressions énergiques devraient être faites pour l'application de la loi. e-Le Crédit ouvrier pour que toutes les familles ouvrières puissent avoir accès à la propriété. Ce sont d'ailleurs les grands désirs du Souverain Pontife manifestés dans les Encycliques. C’est aussi la meilleure manière d'éliminer le terrible problème de l'insécurité dont souffre la classe ouvrière. C'est enfin un remède excellent contre toute doctrine subversive.

Dans une démocratie bien organisée il est de grande importance que les gouvernants et le public 
soient informés de ce que pensent les organisations professionnelles nationales. Parmi les divers groupements qui composent la nation, les associations ouvrières se classent parmi celles qui comprennent leur rôle et qui se font un devoir d'étudier les divers problèmes de l'heure, tant au point de vue syndical, que social et d'intérêt général, de les discuter et d'en faire part.

Avec la guerre et les développements de la civilisation actuelle, les Syndicats pénètrent de plus en plus dans la vie de la communauté nanationale. Dans l'ordre social comme économique, rien n'est plus résolu sans le concours des syndicats ouvriers. L'évolution du syndicalisme, en ce sens, ne peut étonner personne car il faut avoir à l'esprit que le but primordial du mouvement syndical est d'étudier, de promouvoir et de défendre les intérêts professionnels des travailleurs. Il faut reconnaître dans leurs revendications la poussée d'une force qui monte et qui de par sa nature mê- me doit être intégrée à la vie de la nation.

Nous regrettons de ne pas avoir le compterendu de la convention du Congrès canadien du travail qui représente lui aussi les aspirations d'un grand nombre d'ouvriers canadiens. ${ }^{4}$

Malgré les divergences d'inspiration et de technique d'action, il est à remarquer que nos divers groupes d'ouvriers syndiqués s'entendent sur plusieurs points. S'il en est ainsi, c'est donc dire que ce sont là des besoins très importants dont devraient tenir compte les chefs d'Etat, les chefs d'entreprises et le public pour mieux comprendre les réelles aspirations de la classe ouvrière. Les autres groupements professionnels devraient en faire autant. Ce serait vraiment là la démocratie en oeuvre.

(4) Toujours d'après les mêmes statistiques le Congrès canadien du Travail compte 314,025 membres répartis en 338 sections locales.

\section{ETHICS IN LABOUR RELATIONS}

\section{Gaston CHOLETTE}

To the ordinary bystander who witnesses the evolution of labour relations, the latter seem to consist of a mere series of endless disputes and struggles. However, in reality such is not the fact since in spite of the numerous disputes and sensational developments to which much publicity is given there are fortunately more and more cases of fruitful co-operation and mutual understanding that are worthy of being noted. Nevertheless, despite the endeavours of the religious and edifying influences among the Catholic associations grouping employers and workers, the ideals of justice and charity that should prevail in the economic field in this Province are still far from being reached.

Although labour legislation's purpose is to furnish associations with a status guaranteeing the existence and main features of its functions, as also to ensure the harmonious co-operation of the parties in the settlement of disputes and the negotiation of collective agreements, such legislation is far from proving entirely satisfactory and from establishing social peace by the mere fact of its existence. Many people even claim that certain parts of this legislation obstruct the progress of justice, as the mode of procedure prescribed thereby appears to them as constituting a series of difficulties which might sometimes be used to com- plicate the enforcement and interpretation of the labour laws in existence and to provoke the workers by delaying agreements systematically.

Would it be be preferable to ameliorate this legislation? Obviously, indeed, because it often happens that the power of the law is the only thing capable of bringing certain people to reason. However, does not everyday experience with the labour laws in existence tend to prove that one must not entirely rely on them to obtain satisfactory results, even after such laws have been mended or ameliorated ? Quid leges sine moribus: what are laws worth without morals? The principal «social encyclicals», namely : Quadragesimo Anno and Divini Redemptoris, are so explicit that no Catholic may entertain doubts regarding this question.

Morality : ethics! That is the supreme corrective to the great unrest in industrial relations. As a matter of fact, morality is a term that applies not only to the virtues of temperance and chastity, to the sixth and ninth Commandments, but also to all the other virtues and deeds of man. Morality is therefore relevant to the great social virtues: justice and charity. Then, it is an error to limit the meaning of the word morality so that it may only apply to questions of purity.

Let us now see the manner in which morality 